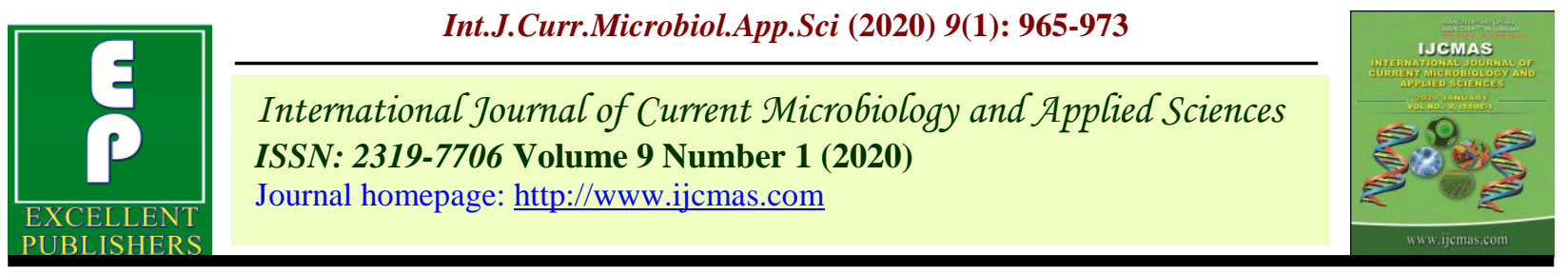

Original Research Article

https://doi.org/10.20546/ijcmas.2020.901.108

\title{
Effect of Pruning Intensity and Foliar Feeding of Nutrients on Growth and Quality of Phalsa (Grewia subinaeqalis D.C)
}

\author{
H. K. Singh ${ }^{1 *}$, Shashank Singh ${ }^{1}$, Ashish $\operatorname{Singh}^{2}$, Bhanu Pratap ${ }^{1}$ and Anil Kumar \\ ${ }^{1}$ Department of Horticulture, NDUAT, Ayodhya, (U.P.) 224229, India \\ ${ }^{2}$ Department of Horticulture, JS University, Shikohabad, (U.P.) 283135, India \\ ${ }^{3}$ Department of Horticulture, SHUATS, Prayagraj (U.P.) 211007, India \\ *Corresponding author
}

\section{A B S T R A C T}

\section{Keywords}

Pruning Intensity,

Urea, $\mathrm{ZnSO}_{4}$,

$\mathrm{K}_{2} \mathrm{SO}_{4}$ and Phalsa

Article Info

Accepted:

15 December 2019

Available Online:

20 January 2020
A field experiment was conducted at Main Experiment Station, Department of Horticulture, NDUAT, Kumarganj, Faizabad (U.P.). Pruning of Phalsa at $50 \mathrm{~cm}$ above ground level combined with $\mathrm{ZnSO}_{4} \quad 0.4 \%$ increased number of shoot and fruiting nodes closely followed by Urea $2 \%$ and $\mathrm{K}_{2} \mathrm{SO}_{4}$ $0.2 \%$ with pruning at $50 \mathrm{~cm}$ above ground level. Better response was obtained with fruit yield attributing attributes also. The fruit quality in terms of TSS, acidity, ascorbic acid content, juice percent and sugar content were better with $\mathrm{ZnSO}_{4} 0.4 \%$ combined with pruning at $50 \mathrm{~cm}$ above ground level closely followed by $\mathrm{K}_{2} \mathrm{SO}_{4} 0.2 \%$ with pruning at $50 \mathrm{~cm}$ above

\section{Introduction}

Phalsa is also known as star apple, a subtropical fruit which is cultivated commercially in various states of India like Punjab, Haryana, Uttar Pradesh and Madhya Pradesh. Phalsa is also cultivated in limited scale in the states of Maharashtra, Gujrat, Andhra Pradesh, Bihar and West Bengal. The fruits of phalsa are eaten fresh. The juice of phalsa is used for making squash and syrup. Since vedic times phalsa is known for its good medicinal properties. The woody part of phalsa is used for fuel by rural people and is also used for basket making which is an income generating entrepreneurship to the rural farmers and poor people. It is quick growing, very hardly shrub which thrives well in wide range of soils even on salt affected wastelands. Phalsa can also be grown as intercrop in mango, aonla, bael and ber orchards. In India, the phalsa grows well up to an elevation of 3,000 ft (914 m). It can stand light frosts which cause only shedding of leaves. The phalsa grows in most any soilsandy, clay or limestone but rich loam improves fruit production, along with irrigation during the fruiting season and in dry 
periods, even though the tree is droughttolerant. Generally, it is grown in marginal land close to city markets. Seeds are the usual means of propagation and they germinate in 15 days. Ground-layers, treated with hormones, have been $50 \%$ successful; airlayers, $85 \%$. Cuttings are difficult to root. Only $20 \%$ of semi-hardwood cuttings from spring flush, treated with 1,000 ppm NAA, and planted in July (in India) rooted and grew normally. The flower contains grewinol, a long chain keto alcohol (Laxmi and Chauhan, 1976). The seed of phalsa contains $5 \%$ oil, which is bright yellow in colour and contains $65 \%$ linoleic acid, $13.5 \%$ oleic acid and $11 \%$ stearic acid (Morton, 1987).

Little or meager information is available on effect of pruning combined with foliar feeding for better plant growth and higher production of quality fruits of this crop.

\section{Materials and Methods}

Field experiment was conducted at Main Experiment Station, Department of Horticulture, NDUAT, Kumarganj, Faizabad (U.P.). Forty five plants of eighteen years old phalsa cv. Sharbati, uniform in size and vigour were planted at distance at $3 \times 2 \mathrm{~m}$ were selected. The experiment was laid out in Factorial RBD with three replications having 15 treatment combination including 3 levels of pruning viz. pruned at $25 \mathrm{~cm}, 50 \mathrm{~cm}$ and 75 $\mathrm{cm}$ above ground level and 5 chemical treatment viz. control (water), $\mathrm{ZnSO}_{4} 0.4 \%$, $\mathrm{CuSO}_{4} \quad 0.4 \%, \mathrm{~K}_{2} \mathrm{SO}_{4} \quad 0.2 \%$ and urea $2 \%$ solution.

Pruning of phalsa bushes was done in first week of February and spray of nutrient solution was done on second fortnight of March (pre $100 \mathrm{~m}$ stage) while second spray was done after fruit setting. Observations were recorded on number of shoots/fruiting nodes, fruits/node, fruit yield/plant, weight of 50 fruits, juice percent and quality of juice in terms of TSS, acidity ascorbic acid and sugar content as per methods given in A.O.A.C (1996).

Foliar treatments: $\mathrm{C}_{0^{-}}$Control (water); $\mathrm{C}_{1^{-}}$ $\mathrm{ZnSO}_{4} \quad 0.4 \% ; \mathrm{C}_{2}-\mathrm{CuSO}_{4} \quad 0.4 \% ; \mathrm{C}_{3}-\mathrm{K}_{2} \mathrm{SO}_{4}$ $0.4 \% ; \mathrm{C}_{4}$-Urea $2.0 \%$.

Pruning intensity: $\mathrm{P}_{1^{-}}-25 \mathrm{~cm} ; \mathrm{P}_{2^{-}} 50 \mathrm{~cm}$ and $\mathrm{P}_{3^{-}}-75 \mathrm{~cm}$.

\section{Results and Discussion}

A perusal of data presented in Table 1 (a and b) reveals that shoot per plant, fruiting node per shoot, fruits/node and fruit yield was influenced significantly. Pruning intensity and foliar feeding of nutrients influenced all yield characters significantly. The highest number of shoots (300), nodes/shoot (18.08), fruit number per were recorded with pruning 50 $\mathrm{cm}$ above ground level. Same level of pruning and foliar feeding of $\mathrm{ZnSO}_{4} \quad 0.4 \%$ for followed by urea $2.0 \%$. The increase in growth parameters might be due to the fact that Potash, Zinc and Copper activate many enzymes required for photosynthesis and also plays a vital role in metabolism and same time spray of and Urea is constituent of protein essential for formation of protoplasm, influence cell division, elongation and causes better plant growth. The findings are in line with the findings of (Kumar et al., 2004) in litchi and (Kumar et al., 2008 and Rathore, 2010) in phalsa.

It is also clear from the data (Table 2a) that response of pruning and nutrients also increased fruit yield being highest $(4.10 \mathrm{~kg}$ per plant) with pruning $50 \mathrm{~cm}$ above ground level combined with $\mathrm{ZnSO}_{4} \quad 0.4 \%$ foliar feeding follow by same level of pruning follow by spray of Urea $2.0 \%$. Similar findings were observed by Tiwari et al., (2011) in aonla and Mishra and Pathak (1998) in guava. 
A perusal of data presented in table 2 (a and b) that weight of 50 fruit and juice percent were influenced by pruning intensity and feeding of nutrients. The highest 50 fruits was recorded $(47.67 \mathrm{~g})$ pruning at $50 \mathrm{~cm}$ above ground level with $\mathrm{ZnSO}_{4} \quad 0.4 \%$ followed by same level of pruning sprayed with $2.0 \%$ urea foliar feeding followed by $\mathrm{K}_{2} \mathrm{SO}_{4} \quad 0.2 \%$ foliar feeding (49.67) and pruning at $50 \mathrm{~cm}$ above ground level. The present findings are in close agreement with Arora and Yamdagni (1985) in sweet lime, Singh and Singh (2008) in aonla cv. NA-7 and Singh et al., (2001) in aonla cv. Francis where the juice content increased due to fact that nutrients $(\mathrm{N}, \mathrm{K}, \mathrm{Zn}$ and $\mathrm{Cu}$ ) application. Uptake of nutrients in plant with minerals led to increase in turgor pressure which resulted in increased juice percent. The present findings are also in agreement with Kumar (2004) in litchi.

Table.1(a) Effect of pruning intensity and foliar feeding of nutrients on number of shoots per plant

\begin{tabular}{|c|c|c|c|c|}
\hline \multirow[t]{2}{*}{ Treatments } & \multicolumn{3}{|c|}{ Pruning (Above ground level) } & \multirow[t]{2}{*}{ Mean } \\
\hline & $25 \mathrm{~cm}\left(\mathbf{P}_{1}\right)$ & $50 \mathrm{~cm}\left(\mathbf{P}_{2}\right)$ & $75\left(\mathbf{P}_{3}\right)$ & \\
\hline Control (water) $\left(\mathbf{C}_{0}\right)$ & 22.67 & 26.67 & 21.00 & 23.44 \\
\hline $\mathrm{ZnSO}_{4} 0.4 \%\left(\mathrm{C}_{1}\right)$ & 32.00 & 33.33 & 31.33 & 32.22 \\
\hline $\mathrm{CuSO}_{4} 0.4 \%\left(\mathrm{C}_{2}\right)$ & 29.00 & 29.67 & 28.67 & 29.11 \\
\hline $\mathrm{K}_{2} \mathrm{SO}_{4} 0.4 \%\left(\mathrm{C}_{3}\right)$ & 30.00 & 30.33 & 29.67 & 30.00 \\
\hline Urea $2.0 \%\left(C_{4}\right)$ & 32.67 & 33.00 & 30.67 & 32.11 \\
\hline \multirow[t]{2}{*}{ Mean } & 29.27 & 30.60 & 28.27 & \\
\hline & $\mathbf{P}$ & $\mathbf{C}$ & \multicolumn{2}{|c|}{ PxC } \\
\hline SEm \pm & 0.40 & 0.51 & \multicolumn{2}{|c|}{$\mathbf{0 . 8 9}$} \\
\hline CD at $5 \%$ & 1.15 & 1.48 & \multicolumn{2}{|c|}{ NS } \\
\hline
\end{tabular}

Table.1(b) Effect of pruning intensity and foliar feeding of nutrients number of fruiting nodes per shoot

\begin{tabular}{|c|c|c|c|c|}
\hline \multirow[t]{2}{*}{ Treatments } & \multicolumn{3}{|c|}{ Pruning (Above ground level) } & \multirow[t]{2}{*}{ Mean } \\
\hline & $25 \mathrm{~cm}\left(\mathbf{P}_{1}\right)$ & $50 \mathrm{~cm}\left(\mathbf{P}_{2}\right)$ & $75\left(\mathbf{P}_{3}\right)$ & \\
\hline Control (water) $\left(\mathbf{C}_{0}\right)$ & 15.67 & 16.40 & 14.67 & 15.58 \\
\hline $\mathrm{ZnSO}_{4} 0.4 \%\left(\mathrm{C}_{1}\right)$ & 18.67 & 19.80 & 18.33 & 18.93 \\
\hline $\mathrm{CuSO}_{4} 0.4 \%\left(\mathrm{C}_{2}\right)$ & 17.00 & 17.60 & 16.67 & 17.09 \\
\hline $\mathrm{K}_{2} \mathrm{SO}_{4} 0.4 \%\left(\mathrm{C}_{3}\right)$ & 16.67 & 18.00 & 17.33 & 17.33 \\
\hline Urea $2.0 \%\left(C_{4}\right)$ & 17.67 & 18.60 & 16.00 & 17.42 \\
\hline \multirow[t]{2}{*}{ Mean } & 17.14 & 18.08 & 16.60 & \\
\hline & $\mathbf{P}$ & $\mathbf{C}$ & \multicolumn{2}{|c|}{ PxC } \\
\hline SEm \pm & 0.31 & 0.40 & \multicolumn{2}{|c|}{0.70} \\
\hline CD at $5 \%$ & 0.90 & 1.17 & \multicolumn{2}{|c|}{2.02} \\
\hline
\end{tabular}


Table.1(c) Effect of pruning intensity and foliar feeding of nutrients on number of fruits per node

\begin{tabular}{|c|c|c|c|c|}
\hline \multirow[t]{2}{*}{ Treatments } & \multicolumn{3}{|c|}{ Pruning (Above ground level) } & \multirow[t]{2}{*}{ Mean } \\
\hline & $25 \mathrm{~cm}\left(\mathbf{P}_{1}\right)$ & $50 \mathrm{~cm}\left(P_{2}\right)$ & $75\left(\mathbf{P}_{3}\right)$ & \\
\hline Control (water) $\left(\mathbf{C}_{0}\right)$ & 14.00 & 14.70 & 14.33 & 14.34 \\
\hline $\mathrm{ZnSO}_{4} \mathbf{0 . 4 \%}\left(\mathrm{C}_{1}\right)$ & 16.33 & 17.15 & 16.00 & 16.49 \\
\hline $\mathrm{CuSO}_{4} \mathbf{0 . 4 \%}\left(\mathrm{C}_{2}\right)$ & 15.33 & 16.10 & 15.00 & 15.48 \\
\hline $\mathrm{K}_{2} \mathrm{SO}_{4} 0.4 \%\left(\mathrm{C}_{3}\right)$ & 15.67 & 16.40 & 14.67 & 15.58 \\
\hline Urea $2.0 \%\left(C_{4}\right)$ & 16.33 & 17.10 & 15.00 & 16.14 \\
\hline \multirow[t]{2}{*}{ Mean } & 1553 & 16.29 & 15.00 & \\
\hline & $\mathbf{P}$ & C & \multicolumn{2}{|c|}{ PxC } \\
\hline SEm \pm & 0.28 & 0.37 & \multicolumn{2}{|c|}{0.63} \\
\hline CD at $5 \%$ & 0.82 & 1.06 & \multicolumn{2}{|c|}{1.84} \\
\hline
\end{tabular}

Table.1(d) Effect of pruning intensity and foliar feeding of nutrients on fruit yield per plant (kg/plant)

\begin{tabular}{|c|c|c|c|c|}
\hline \multirow[t]{2}{*}{ Treatments } & \multicolumn{3}{|c|}{ Pruning (Above ground level) } & \multirow[t]{2}{*}{ Mean } \\
\hline & $25 \mathrm{~cm}\left(\mathbf{P}_{1}\right)$ & $50 \mathrm{~cm}\left(\mathbf{P}_{2}\right)$ & $75\left(\mathbf{P}_{3}\right)$ & \\
\hline Control (water) $\left(C_{0}\right)$ & 2.84 & 3.10 & 2.83 & 2.92 \\
\hline $\mathrm{ZnSO}_{4} \mathbf{0 . 4 \%}\left(\mathrm{C}_{1}\right)$ & 3.70 & 4.10 & 3.67 & 3.82 \\
\hline $\mathrm{CuSO}_{4} \mathbf{0 . 4 \%}\left(\mathrm{C}_{2}\right)$ & 3.60 & 3.95 & 3.63 & 3.73 \\
\hline $\mathrm{K}_{2} \mathrm{SO}_{4} \mathbf{0 . 4 \%}\left(\mathrm{C}_{3}\right)$ & 3.56 & 3.90 & 3.79 & 3.81 \\
\hline Urea $2.0 \%\left(C_{4}\right)$ & 3.68 & 4.05 & 3.71 & \\
\hline \multirow[t]{2}{*}{ Mean } & 3.48 & 3.82 & 3.53 & \\
\hline & $\mathbf{P}$ & $\mathbf{C}$ & \multicolumn{2}{|c|}{$\mathbf{P x C}$} \\
\hline SEm \pm & 0.07 & 0.08 & \multicolumn{2}{|c|}{0.15} \\
\hline CD at $5 \%$ & 0.19 & 0.25 & \multicolumn{2}{|c|}{0.43} \\
\hline
\end{tabular}

Table.1(e) Effect of pruning intensity and foliar feeding of nutrients on number of fruiting nodes per shoot

\begin{tabular}{|c|c|c|c|c|}
\hline \multirow[t]{2}{*}{ Treatments } & \multicolumn{3}{|c|}{ Pruning (Above ground level) } & \multirow[t]{2}{*}{ Mean } \\
\hline & $25 \mathrm{~cm}\left(\mathbf{P}_{1}\right)$ & $50 \mathrm{~cm}\left(\mathbf{P}_{2}\right)$ & $75\left(P_{3}\right)$ & \\
\hline Control (water) $\left(C_{0}\right)$ & 15.67 & 16.40 & 14.67 & 15.58 \\
\hline $\mathrm{ZnSO}_{4} \mathbf{0 . 4 \%}\left(\mathrm{C}_{1}\right)$ & 18.67 & 19.80 & 18.33 & 18.93 \\
\hline $\mathrm{CuSO}_{4}$ 0.4\% $\left(\mathrm{C}_{2}\right)$ & 17.00 & 17.60 & 16.67 & 17.09 \\
\hline $\mathrm{K}_{2} \mathrm{SO}_{4} \mathrm{O.4 \%}\left(\mathrm{C}_{3}\right)$ & 16.67 & 18.00 & 17.33 & 17.33 \\
\hline Urea $2.0 \%\left(C_{4}\right)$ & 17.67 & 18.60 & 16.00 & 17.42 \\
\hline \multirow[t]{2}{*}{ Mean } & 17.14 & 18.08 & 16.60 & \\
\hline & $\mathbf{P}$ & C & \multicolumn{2}{|c|}{ PxC } \\
\hline SEm \pm & 0.31 & 0.40 & \multicolumn{2}{|c|}{0.70} \\
\hline CD at $5 \%$ & 0.90 & 1.17 & \multicolumn{2}{|c|}{2.02} \\
\hline
\end{tabular}


Table.2(a) Effect of pruning intensity and foliar feeding of nutrients on weight of 50 fruits (gm)

\begin{tabular}{|c|c|c|c|c|}
\hline \multirow[t]{2}{*}{ Treatments } & \multicolumn{3}{|c|}{ Pruning (Above ground level) } & \multirow[t]{2}{*}{ Mean } \\
\hline & $25 \mathrm{~cm}\left(\mathbf{P}_{1}\right)$ & $50 \mathrm{~cm}\left(P_{2}\right)$ & $75\left(\mathbf{P}_{3}\right)$ & \\
\hline Control (water) $\left(\mathbf{C}_{0}\right)$ & 42.67 & 43.00 & 42.33 & 42.67 \\
\hline $\mathrm{ZnSO}_{4} 0.4 \%\left(\mathrm{C}_{1}\right)$ & 47.00 & 47.67 & 42.00 & 45.56 \\
\hline $\mathrm{CuSO}_{4} 0.4 \%\left(\mathrm{C}_{2}\right)$ & 40.33 & 42.00 & 41.00 & 41.11 \\
\hline $\mathrm{K}_{2} \mathrm{SO}_{4} 0.4 \%\left(\mathrm{C}_{3}\right)$ & 43.00 & 44.33 & 41.33 & 42.89 \\
\hline Urea $2.0 \%\left(C_{4}\right)$ & 43.33 & 44.00 & 43.00 & 43.44 \\
\hline \multirow[t]{2}{*}{ Mean } & 43.27 & 44.20 & 41.93 & \\
\hline & $\mathbf{P}$ & $\mathbf{C}$ & \multicolumn{2}{|c|}{ PxC } \\
\hline SEm \pm & 0.27 & 0.35 & \multicolumn{2}{|c|}{0.61} \\
\hline CD at $5 \%$ & 0.79 & 1.01 & \multicolumn{2}{|c|}{1.76} \\
\hline
\end{tabular}

Table.2(b) Effect of pruning intensity and foliar feeding of nutrients on juice percent (\%)

\begin{tabular}{|c|c|c|c|c|}
\hline \multirow[t]{2}{*}{ Treatments } & \multicolumn{3}{|c|}{ Pruning (Above ground level) } & \multirow[t]{2}{*}{ Mean } \\
\hline & $25 \mathrm{~cm}\left(\mathbf{P}_{1}\right)$ & $50 \mathrm{~cm}\left(\mathbf{P}_{2}\right)$ & $75\left(\mathbf{P}_{3}\right)$ & \\
\hline Control (water) $\left(\mathbf{C}_{0}\right)$ & 37.00 & 37.00 & 36.33 & 36.78 \\
\hline $\mathrm{ZnSO}_{4} \mathbf{0 . 4 \%}\left(\mathrm{C}_{1}\right)$ & 47.67 & 48.67 & 44.67 & 47.00 \\
\hline $\mathrm{CuSO}_{4} \mathbf{0 . 4 \%}\left(\mathrm{C}_{2}\right)$ & 46.67 & 50.33 & 43.33 & 46.78 \\
\hline $\mathrm{K}_{2} \mathrm{SO}_{4} 0.4 \%\left(\mathrm{C}_{3}\right)$ & 50.00 & 51.00 & 48.00 & 49.67 \\
\hline Urea $2.0 \%\left(\mathrm{C}_{4}\right)$ & 49.33 & 52.00 & 49.00 & 50.11 \\
\hline \multirow[t]{2}{*}{ Mean } & 46.13 & 47.80 & 44.27 & \\
\hline & $\mathbf{P}$ & $\mathbf{C}$ & \multicolumn{2}{|c|}{ PxC } \\
\hline SEm \pm & 0.47 & 0.61 & \multicolumn{2}{|c|}{1.05} \\
\hline CD at $5 \%$ & 1.36 & 1.76 & \multicolumn{2}{|c|}{3.04} \\
\hline
\end{tabular}

Table.3(a) Effect of pruning intensity and foliar feeding of nutrients on fruit quality (TSS)

\begin{tabular}{|c|c|c|c|c|}
\hline \multirow[t]{2}{*}{ Treatments } & \multicolumn{3}{|c|}{ Pruning (Above ground level) } & \multirow[t]{2}{*}{ Mean } \\
\hline & $25 \mathrm{~cm}\left(\mathbf{P}_{1}\right)$ & $50 \mathrm{~cm}\left(P_{2}\right)$ & $75\left(\mathbf{P}_{3}\right)$ & \\
\hline Control (water) $\left(\mathbf{C}_{0}\right)$ & 19.22 & 21.23 & 18.38 & 19.61 \\
\hline $\mathrm{ZnSO}_{4} 0.4 \%\left(\mathrm{C}_{1}\right)$ & 27.65 & 28.40 & 26.76 & 27.87 \\
\hline $\mathrm{CuSO}_{4} 0.4 \%\left(\mathrm{C}_{2}\right)$ & 26.12 & 26.34 & 25.16 & 25.87 \\
\hline $\mathrm{K}_{2} \mathrm{SO}_{4} 0.4 \%\left(\mathrm{C}_{3}\right)$ & 26.91 & 27.62 & 25.82 & 26.78 \\
\hline Urea $2.0 \%\left(\mathrm{C}_{4}\right)$ & 26.13 & 27.13 & 25.87 & 26.38 \\
\hline \multirow[t]{2}{*}{ Mean } & 25.20 & 26.15 & 24.40 & \\
\hline & $\mathbf{P}$ & $\mathbf{C}$ & \multicolumn{2}{|c|}{ PxC } \\
\hline SEm \pm & 0.19 & 0.25 & \multicolumn{2}{|c|}{0.43} \\
\hline CD at $5 \%$ & 0.56 & 0.72 & \multicolumn{2}{|c|}{1.25} \\
\hline
\end{tabular}


Table.3(b) Effect of pruning intensity and foliar feeding of nutrients on acidity (\%)

\begin{tabular}{|c|c|c|c|c|}
\hline \multirow[t]{2}{*}{ Treatments } & \multicolumn{3}{|c|}{ Pruning (Above ground level) } & \multirow[t]{2}{*}{ Mean } \\
\hline & $25 \mathrm{~cm}\left(\mathbf{P}_{1}\right)$ & $50 \mathrm{~cm}\left(\mathbf{P}_{2}\right)$ & $75\left(P_{3}\right)$ & \\
\hline Control (water) $\left(C_{0}\right)$ & 2.35 & 2.20 & 2.30 & 2.28 \\
\hline $\mathrm{ZnSO}_{4} \mathbf{0 . 4 \%}\left(\mathrm{C}_{1}\right)$ & 1.83 & 1.80 & 1.88 & 1.84 \\
\hline $\mathrm{CuSO}_{4} \mathbf{0 . 4 \%}\left(\mathrm{C}_{2}\right)$ & 2.25 & 2.32 & 2.27 & 2.28 \\
\hline $\mathrm{K}_{2} \mathrm{SO}_{4} 0.4 \%\left(\mathrm{C}_{3}\right)$ & 1.96 & 2.02 & 2.05 & 2.01 \\
\hline Urea $2.0 \%\left(C_{4}\right)$ & 2.22 & 2.13 & 2.35 & 2.23 \\
\hline \multirow[t]{2}{*}{ Mean } & 2.12 & 2.09 & 2.17 & \\
\hline & $\mathbf{P}$ & C & \multicolumn{2}{|c|}{ PxC } \\
\hline SEm \pm & 0.03 & 0.04 & \multicolumn{2}{|c|}{0.06} \\
\hline CD at $5 \%$ & NS & 0.10 & \multicolumn{2}{|c|}{ NS } \\
\hline
\end{tabular}

Table.3(c) Effect of pruning intensity and foliar feeding of nutrients on ascorbic acid content (mg/100g pulp)

\begin{tabular}{|c|c|c|c|c|}
\hline \multirow[t]{2}{*}{ Treatments } & \multicolumn{3}{|c|}{ Pruning (Above ground level) } & \multirow{2}{*}{ Mean } \\
\hline & $25 \mathrm{~cm}\left(\mathbf{P}_{1}\right)$ & $50 \mathrm{~cm}\left(\mathbf{P}_{2}\right)$ & $75\left(\mathbf{P}_{3}\right)$ & \\
\hline Control (water) $\left(\mathbf{C}_{0}\right)$ & 27.03 & 27.81 & 26.65 & 27.16 \\
\hline $\mathrm{ZnSO}_{4} \mathbf{0 . 4 \%}\left(\mathrm{C}_{1}\right)$ & 36.50 & 37.28 & 35.98 & 36.59 \\
\hline $\mathrm{CuSO}_{4}$ 0.4\% $\left(\mathrm{C}_{2}\right)$ & 34.86 & 35.58 & 33.97 & $\mathbf{3 4 . 8 0}$ \\
\hline $\mathrm{K}_{2} \mathrm{SO}_{4} \mathbf{0 . 4 \%}\left(\mathrm{C}_{3}\right)$ & 35.90 & 36.63 & 35.05 & 35.86 \\
\hline Urea $2.0 \%\left(C_{4}\right)$ & 35.21 & 35.91 & 34.86 & 35.32 \\
\hline \multirow[t]{2}{*}{ Mean } & 33.90 & 34.64 & 33.30 & \\
\hline & $\mathbf{P}$ & C & \multicolumn{2}{|c|}{ PxC } \\
\hline SEm \pm & 0.22 & 0.29 & \multicolumn{2}{|c|}{0.49} \\
\hline CD at $5 \%$ & 0.64 & 0.83 & \multicolumn{2}{|c|}{ NS } \\
\hline
\end{tabular}

Table.4(a) Effect of pruning intensity and foliar feeding of nutrients on reducing sugar (\%)

\begin{tabular}{|c|c|c|c|c|}
\hline \multirow[t]{2}{*}{ Treatments } & \multicolumn{3}{|c|}{ Pruning (Above ground level) } & \multirow[t]{2}{*}{ Mean } \\
\hline & $25 \mathrm{~cm}\left(\mathbf{P}_{1}\right)$ & $50 \mathrm{~cm}\left(\mathbf{P}_{2}\right)$ & $75\left(P_{3}\right)$ & \\
\hline Control (water) $\left(\mathbf{C}_{0}\right)$ & 11.52 & 11.93 & 10.57 & \\
\hline $\mathrm{ZnSO}_{4} \mathbf{0 . 4 \%}\left(\mathrm{C}_{1}\right)$ & 13.29 & 13.76 & 13.25 & \\
\hline $\mathrm{CuSO}_{4}{ }^{0.4 \%}\left(\mathrm{C}_{2}\right)$ & 12.88 & 13.07 & 12.48 & \\
\hline $\mathrm{K}_{2} \mathrm{SO}_{4} 0.4 \%\left(\mathrm{C}_{3}\right)$ & 12.92 & 13.13 & 12.73 & \\
\hline Urea $2.0 \%\left(C_{4}\right)$ & 12.80 & 12.87 & 12.86 & \\
\hline \multirow[t]{2}{*}{ Mean } & 12.68 & 12.95 & 12.37 & \\
\hline & $\mathbf{P}$ & C & \multicolumn{2}{|c|}{ PxC } \\
\hline SEm \pm & 0.15 & 0.19 & \multicolumn{2}{|c|}{0.33} \\
\hline CD at $5 \%$ & 0.43 & 0.56 & \multicolumn{2}{|c|}{ NS } \\
\hline
\end{tabular}


Table.4(b) Effect of pruning intensity and foliar feeding of nutrients on non-reducing sugar (\%)

\begin{tabular}{|c|c|c|c|c|}
\hline \multirow[t]{2}{*}{ Treatments } & \multicolumn{3}{|c|}{ Pruning (Above ground level) } & \multirow[t]{2}{*}{ Mean } \\
\hline & $25 \mathrm{~cm}\left(\mathbf{P}_{1}\right)$ & $50 \mathrm{~cm}\left(\mathbf{P}_{2}\right)$ & $75\left(\mathbf{P}_{3}\right)$ & \\
\hline Control (water) $\left(\mathbf{C}_{\mathbf{0}}\right)$ & 3.16 & 3.27 & 2.90 & 3.11 \\
\hline $\mathrm{ZnSO}_{4} 0.4 \%\left(\mathrm{C}_{1}\right)$ & 3.65 & 3.77 & 3.63 & 3.69 \\
\hline $\mathrm{CuSO}_{4} 0.4 \%\left(\mathrm{C}_{2}\right)$ & 3.53 & 3.58 & 3.42 & 3.51 \\
\hline $\mathrm{K}_{2} \mathrm{SO}_{4} 0.4 \%\left(\mathrm{C}_{3}\right)$ & 3.54 & 3.60 & 3.49 & 3.55 \\
\hline Urea $2.0 \%\left(C_{4}\right)$ & 3.51 & 3.53 & 3.53 & 3.52 \\
\hline \multirow[t]{2}{*}{ Mean } & 3.48 & 3.55 & 3.40 & \\
\hline & $\mathbf{P}$ & $\mathbf{C}$ & \multicolumn{2}{|c|}{ PxC } \\
\hline SEm \pm & 0.04 & 0.05 & \multicolumn{2}{|c|}{0.09} \\
\hline CD at $5 \%$ & 0.12 & 0.15 & \multicolumn{2}{|c|}{ NS } \\
\hline
\end{tabular}

Table.4(c) Effect of pruning intensity and foliar feeding of nutrients total sugars (\%)

\begin{tabular}{|c|c|c|c|c|}
\hline \multirow[t]{2}{*}{ Treatments } & \multicolumn{3}{|c|}{ Pruning (Above ground level) } & \multirow[t]{2}{*}{ Mean } \\
\hline & $25 \mathrm{~cm}\left(\mathbf{P}_{1}\right)$ & $50 \mathrm{~cm}\left(P_{2}\right)$ & $75\left(\mathbf{P}_{3}\right)$ & \\
\hline Control (water) $\left(\mathbf{C}_{0}\right)$ & 14.68 & 15.20 & 13.47 & 14.45 \\
\hline $\mathrm{ZnSO}_{4} 0.4 \%\left(\mathrm{C}_{1}\right)$ & 16.94 & 17.53 & 16.88 & 17.12 \\
\hline $\mathrm{CuSO}_{4} 0.4 \%\left(\mathrm{C}_{2}\right)$ & 16.42 & 16.65 & 15.90 & 16.32 \\
\hline $\mathrm{K}_{2} \mathrm{SO}_{4} 0.4 \%\left(\mathrm{C}_{3}\right)$ & 16.46 & 16.73 & 16.22 & 16.47 \\
\hline Urea $2.0 \%\left(\mathrm{C}_{4}\right)$ & 16.32 & 16.40 & 16.38 & 16.37 \\
\hline \multirow[t]{2}{*}{ Mean } & 16.16 & 16.50 & 15.77 & \\
\hline & $\mathbf{P}$ & $\mathbf{C}$ & \multicolumn{2}{|c|}{ PxC } \\
\hline SEm \pm & 0.19 & 0.25 & \multicolumn{2}{|c|}{0.43} \\
\hline CD at $5 \%$ & 0.56 & 0.71 & \multicolumn{2}{|c|}{1.25} \\
\hline
\end{tabular}

Pruning intensity and foliar feeding of nutrients influenced the TSS, acidity and ascorbic acid content in juice Table 1 (a and b). The interaction effect between pruning levels and chemicals on TSS was found significant and noted highest (28.40) with combined effect of $\mathrm{ZnSO}_{4} 0.4 \%$ along with pruning at $50 \mathrm{~cm}$ above ground level and effect was significant than rest of the combination. The increase in TSS content may be explained by the fact that applied nutrients are helpful to photosynthesis which ultimately led to the accumulation of carbohydrate which helps to increase TSS content of juice. The acid content in juice decreased significantly with foliar feeding, while non-significant effect was observed with pruning levels. The reason for decreased acidity due to nutrients $(\mathrm{N}, \mathrm{K}, \mathrm{Zn}$ and $\mathrm{Cu}$ ) might be due to increase in translocation of carbohydrates due to conversion of acid to sugar. These findings are in agreement to Joon et al., (1984). The highest ascorbic acid content $(37.28 \mathrm{mg} / 100 \mathrm{~g}$ juice) was recorded with combined effect of $\mathrm{ZnSO}_{4} \quad 0.4 \%$ closely followed by spray of $\mathrm{K}_{2} \mathrm{SO}_{4} \quad 0.2 \%$ (36.63 $\mathrm{mg} / 100 \mathrm{~g}$ juice) along with pruning at $50 \mathrm{~cm}$ above ground level. Similar response has also been reported by Singh et al., (2009) in aonla and Singh et al., (1995) in ber. The increase in ascorbic acid content may be attributed to quality improving properties of $\mathrm{N}, \mathrm{K}, \mathrm{Zn}$ and $\mathrm{Cu}$. The increase in ascorbic acid content in potassium treated fruits might be due to the 
stimulated function of enzymes which participate in the synthesis of ascorbic acid. The reducing sugar, non-reducing sugar and total sugars in fruits juice of phalsa have also been increased as influenced by pruning levels and spray of nutrients Table 4 (a and b).

The highest level of reducing sugars (13.76), non-reducing sugar $(3.77 \%)$ and total sugar (17.53) were found with foliar spray of $\begin{array}{llllll}\mathrm{ZnSO}_{4} & 0.4 \% & \text { followed by } \mathrm{K}_{2} & \mathrm{SO}_{4} & 0.2 \%\end{array}$ along with pruning at $50 \mathrm{~cm}$ above ground level. The significant increase in sugar content might be due to accumulation of carbohydrates in fruits as a result of $\mathrm{N}, \mathrm{K}, \mathrm{Zn}$ and $\mathrm{Cu}$ application and also the role played by regular pruning of phalsa, as phalsa needs regular pruning of previous season shoots. Similar findings were also reported by Singh et al., (1979) in grapes and Bhatia and Yadav (2005) in ber.

\section{Acknowledgement}

The author acknowledges the institutional support to the present research work.

\section{References}

A.O.A.C (1990). Official METHODS of Analysis Associated Official CHEMIST (12 ${ }^{\text {th }}$ Ed.) Washington D.C.

Aror, R.K. and Yamdagni, R.(1985). Effect of different levels on pruning of flowering, fruit set, final retention and fruit quality in sweetlime (Citrus limettoides Tanaka). Prog. Hort., 17(1): 1-4.

Bhatia, B.S. and Yadav, P.K. (2005). Effect of foiar application of urea and NAA on fruit yield and quality of ber (Zizyphus mauritiana) Nat. Sem. Commercialization of Horticulture in Non-traditional areas. Organized by CIAH, Bikaner (Rajasthan) from s:119.

Joon, M.S., Singh, R.R. and Daulta, B.S.
(1984). Effect of foliar spray of zinc and urea on yield and physico-chemical composition of ber fruit cv. Gala. Haryana J. Sci., 1: 110-112.

Kumar, S., Kumar, S. and Verma, D.K. (2004). Effect of micronutrients and NAA on yield and quality of litchi (Litchi chinesis Garttn. Sonn) cv. Dehradun fruits Abstr: In Proc. Of International Seminar on Rec. Trend in Hi. Tech. Hort. and Pht.,Organized by CSAUAT, Kanpur, 4-6:193.

Kumar, P., Tiwari, J.P. and Kumar, R. (2008). Effect of $\mathrm{N}, \mathrm{P} \& \mathrm{~K}$ on fruiting, yield and fruit quality in guava cv. Pant Prabhat. J. Hort. Sci., 3 (1): 43-47.

Lakshmi, V. and Chauhan. J.S. (1976). Grewinol, a keto-alcohol from the flowers of Grewia asiatica. Lloydia. 39:372-374.

Mishra, H.K. and Pathak, R.A. (1998). Effect of shoot pruning in crop regulation in guvava (Psidium guvajava L.) CV.Sardar. Prog. Hort., 30:78-81.

Morton, J.F. (1987). Phalsa. In: Fruits of warm climates. Julia Morton, Miami, FL.

Singh, S.K. and Singh, H.K. (2008). Pruning behavior in aonla (Emblica officinalis Gaertn.) cv. NA-2. Enviroment and Eco., 26(3): 1039-1041.

Singh, J.P., Singh, H.K., Vishvanath and Bhanu Pratap (2009). Response of foliar feeding of nutrients on growth, yield and quality of phalsa (Grewia subinaequalis D.C) fruits. Annals of Hort., 2(1): 69-71.

Singh, S.K. and Singh, H.K. (2008). Pruning behavior in aonla (Emblica officinalis Gaertn) cv . NA-7. Enviroment \& Eco., 25(1): 1039-1041.

Rathore, A.C. (2010). Optimization of canopy management and training system in phalsa (Grewia subinaequalis Lin.) .under semi arid zone. Indian J.Agrofrestry. 12(1):113-115 
Singh, T.P., Yamdagni, R. and Jindal, P.C. (1979). A note on the effect of potassium sprays on quality of grapes cv. Perlette. Haryana J. Sci., 8(34):207-208

Singh, H.K., Shrivastava, A.K., Dwivedi, R. and Kumar, P. (2001). Effect of foliar feeding of micronutrient on plant growth, fruit quality, yield and internal fruit recrosis of aonla (Emblica officinalis Gaertn). Prog. Hort., 33(1): 80-83.
Khan, S., Singh, H.K., Vishwnath and Bhanu Pratap (2009). Effect of foliar application of Micronutrients and thio Urea on growth, fruit yield and quality of aonla (Emblica officinalis Gaertn) cv. NA-6. Annals of Hort,. 2(1):83-85.

Tiwari, Divya, Singh, H.K. and Shukla, A.K. (2009). Effect of Pruning intensity and nutrients of vegetative growth parameters and yield of aonla (Emblica officinalis Gaertn) cv. Francis. Indian J. Arid Hort., 4(2): 65.

\section{How to cite this article:}

Singh, H. K., Shashank Singh, Ashish Singh, Bhanu Pratap and Anil Kumar. 2020. Effect of Pruning Intensity and Foliar Feeding of Nutrients on Growth and Quality of Phalsa (Grewia subinaeqalis D.C). Int.J.Curr.Microbiol.App.Sci. 9(01): 965-973. doi: https://doi.org/10.20546/ijcmas.2020.901.108 